\title{
Evaluation of strategies for confirming Neisseria gonorrhoeae nucleic acid amplification tests
}

Correspondence
Sarah Alexander
sarah.alexander@hpa.org.uk

Received 1 December 2010

Accepted 12 March 2011

\author{
Sarah Alexander, ${ }^{1}$ Filomeno Coelho da Silva, ${ }^{1}$ Rohini Manuel, ${ }^{2}$ \\ Rajesh Varma ${ }^{2}$ and Catherine Ison ${ }^{1}$
}

\author{
${ }^{1}$ Sexually Transmitted Bacteria Reference Laboratory, Health Protection Agency, 61 Colindale \\ Avenue, London NW9 5HT, UK \\ ${ }^{2}$ Barts and The London NHS Trust, The Royal London Hospital, Whitechapel, London E1 1BB, UK
}

\begin{abstract}
The implementation of widespread unselected screening for Neisseria gonorrhoeae in England, using nucleic acid amplification tests (NAATs), has raised concerns regarding the potential increase in misdiagnoses. To increase the positive predictive value, confirmatory testing of positive specimens has been recommended; however, in practice this can be difficult to perform. This study examined the role of two different testing strategies for confirming the $N$. gonorrhoeae status of specimens that had been examined by the ProbeTec Strand Displacement Amplification (SDA) assay (Becton Dickinson). A total of 227 residual clinical specimens in SDA assay collection tubes were sent for confirmatory testing using two different testing approaches: (i) examination using two in-house real-time PCR assays (opa and porA pseudogene) and (ii) examination using the APTIMA Combo 2 (AC2) assay and the APTIMA Monospecific N. gonorrhoeae (AGC) tests (Gen-Probe). Of the 113 SDA-positive specimens (including low positives) examined, $93 \%$ were confirmed as $N$. gonorrhoeae-positive using either one or both real-time PCR assays. In contrast, only $34 \%$ were confirmed using the AC2 and/or the AGC assays. All 114 SDA-negative specimens were confirmed as negative using all four confirmatory tests. Clearly the AC2 and AGC assays cannot reliably be used to confirm residual specimens in SDA assay transportation buffers, due to the incompatibility of different platform chemistries. Although high rates of confirmation ( $93 \%$ ) can be achieved when examining residual SDA assay specimens using independent real-time PCR assays, establishing well-validated in-house real-time PCR assays for diagnostic use is a large undertaking for many primary laboratories and so such tests may be better confined to specialist laboratory services.
\end{abstract}

\section{INTRODUCTION}

An increasing number of laboratories in England and Wales are using dual nucleic acid amplification tests (NAATs) for the combined detection of both chlamydia and Neisseria gonorrhoeae infections (Benzie et al., 2010). $N$. gonorrhoeae NAATs offer a number of advantages over traditional culture methods, including improved patient flow, faster turnaround times and non-invasive sampling. However, the N. gonorrhoeae component of some of the commercially available NAAT platforms has been shown to cross-react with other members of the Neisseria genus (Palmer et al., 2003). The reduced specificity of some $N$. gonorrhoeae NAATs combined with their application in low prevalence populations has raised concerns about patients being misdiagnosed with gonorrhoea (Ison, 2006).

Abbreviations: AC2, APTIMA Combo 2; AGC, APTIMA Monospecific N. gonorrhoeae; NAAT, nucleic acid amplification test; SDA, Strand Displacement Amplification.
The positive predictive value of $N$. gonorrhoeae NAATs can be increased by the introduction of a secondary confirmatory assay which targets a different genetic sequence, and this approach is recommended in low prevalence settings by both the Health Protection Agency and Health Protection Scotland (Whiley et al., 2006; Health Protection Agency, 2010; Dave \& Eastaway, 2007). However, the implementation of confirmatory testing, in reality, can be difficult to achieve because most platforms do not have a second N. gonorrhoeae target (only the GenProbe APTIMA TMA test has this capability). There are currently only a limited number of studies examining the compatibility of the collection buffers between the different dual NAAT platforms, which means that even in laboratories which have access to two different $N$. gonorrhoeae NAAT testing platforms, supplementary testing may still be difficult.

This study sets out to examine the role of two different testing strategies for confirming the N. gonorrhoeae status 
Table 1. Sequences of primer and probes used in the porA and opa real-time PCR assays

\begin{tabular}{|lll|}
\hline Primer/probe & \multicolumn{1}{c|}{ Sequence $\left(\mathbf{5}^{\prime}-\mathbf{3}^{\prime}\right)$} & Reference \\
\hline papTM-P & FAM-CGCCTATACGCCTGCTACTTTCACGC-BHQ1 & Whiley \& Sloots $(2005)$ \\
papTM-F & CAGCATTCAATTTGTTCCGATC & Whiley \& Sloots $(2005)$ \\
papTM-R & GAACTGGTTCATCTGATTACTTTCCA & Whiley \& Sloots $(2005)$ \\
GCopa-LNA & FAM-CCGATATAATC+CGTC+CTTCAA+CATCAG-TAMRA & Tabrizi et al. $(2005)$ \\
GcopaF & TTGAAACACCGCCCGGAA & Tabrizi et al. $(2005)$ \\
GcopaR & TTTCGGCTCCTTATTCGGTTTAA & Tabrizi et al. $(2005)$ \\
RNP-F & AGA TTT GGA CCT GCG AGC G & Chen et al. $(2007)$ \\
RNP-R & GAG CGG CTG TCT CCA CAA GT & Chen et al. $(2007)$ \\
RNP-P & CY5-TT CTG ACC TGA AGG CTC TGC GCG-BHQ3 & Chen et al. $(2007)$ \\
\hline
\end{tabular}

${ }^{*}+=$ LNA (locked nucleic acid).

of specimens that had been examined by the ProbeTec Strand Displacement Amplification (SDA) assay (Becton Dickinson).

\section{METHODS}

Specimens. A total of 227 urine specimens which had been determined to be N. gonorrhoeae-positive (113) and N. gonorrhoeaenegative (114) using the SDA assay at the local primary laboratory were included in this study. Urine specimens were sourced from patients attending genito-urinary medicine clinics, and following testing at the local laboratory the residual clinical specimens in SDA assay collection tubes were sent to the Sexually Transmitted Bacteria Reference Laboratory for confirmatory N. gonorrhoeae testing. Confirmatory testing was performed using two different approaches: (i) examination using two independent in-house real-time PCR assays targeting the porA pseudogene and the opa gene, respectively; and (ii) examination using the APTIMA Combo 2 (AC2) assay and the APTIMA Monospecific N. gonorrhoeae (AGC) tests (Gen-Probe). For the purpose of this study, any specimen which gave a positive result on a minimum of two of the five $N$. gonorrhoeae tests employed was regarded as a true positive.

Independent real-time PCR confirmatory assays. A DNA extraction was performed from $200 \mu$ of each specimen using the MagNA Pure Compact instrument (Nucleic Acid Isolation kit I) (Roche) according to the manufacturer's instructions. Extracted material was then examined using a slightly modified version of two previously published in-house real-time PCR assays which targeted opa and the porA pseudogene, respectively (Whiley \& Sloots, 2005; Tabrizi et al., 2005).

porA pseudogene real-time PCR. Briefly, PCRs were performed in $25 \mu \mathrm{l}$ volumes which contained the following: $4 \mathrm{mM} \mathrm{MgCl}_{2}, 1 \mathrm{U} \mathrm{Taq}$ DNA polymerase (Applied Biosystems), $5 \mu$ l extracted DNA, $250 \mathrm{nM}$ forward and reverse primers and $500 \mathrm{nM}$ probe (Metabion) (Table 1) (Whiley \& Sloots, 2005). Also included within this PCR were $80 \mathrm{mM}$ primers and probe of a previously described internal control which monitors for inhibition and targets the human RNase P gene (Chen et al., 2007). PCR samples were run on a Rotor-Gene 6000 (Qiagen) using temperature cycling conditions of $10 \mathrm{~min}$ at $95{ }^{\circ} \mathrm{C}$, followed by 40 cycles of $95{ }^{\circ} \mathrm{C}$ for $30 \mathrm{~s}$ and $60{ }^{\circ} \mathrm{C}$ for $60 \mathrm{~s}$.

opa gene real-time PCR. PCR was performed using $20 \mu \mathrm{l}$ reactions containing $5 \mu \mathrm{l}$ extracted DNA, $5 \mathrm{mM} \mathrm{MgCl}_{2}, 1 \mathrm{U}$ Taq DNA polymerase (Applied Biosystems), $250 \mathrm{nM}$ forward and reverse primers and $500 \mathrm{nM}$ probe (Table 1) (Tabrizi et al., 2005). PCRs were run on a Rotor-Gene 6000 instrument using temperature cycling conditions of $10 \mathrm{~min}$ at $95{ }^{\circ} \mathrm{C}$, followed by 50 cycles of $95{ }^{\circ} \mathrm{C}$ for $15 \mathrm{~s}$ and $60{ }^{\circ} \mathrm{C}$ for $60 \mathrm{~s}$.

AC2 and AGC tests. Specimens were prepared by transferring $200 \mu \mathrm{l}$ residual clinical specimen in the SDA assay collection buffer into an APTIMA swab collection tube. The specimens were then tested using both the AC2 and AGC tests according to the manufacturer's instructions.

Ethical considerations. All urine specimens used in this study were collected for primary diagnostic use as part of the patients' routine care. Prior to the confirmatory testing of the residual specimen, all samples were blinded and anonymized.

\section{RESULTS}

In this study, 227 residual urine specimens in SDA assay transport buffers were tested at the primary laboratory, and were then forwarded to the Sexually Transmitted Bacteria Reference Laboratory for confirmatory testing using two different approaches. Of the 113 SDA-positive specimens examined, $93 \%(105 / 113)$ could be confirmed as $N$. gonorrhoeae-positive using either one or both real-time PCR assays and were therefore regarded as true positive specimens (Table 2), with $92 \%(104 / 113)$ and $88 \%(98 /$ 113 ) being confirmed by the porA and opa real-time PCRs, respectively. In contrast, only $34 \%(38 / 113)$ of the SDApositive specimens examined could be confirmed using either one or both the Gen-Probe assays, with $24 \%$ (27/ $113)$ and $27 \%(30 / 113)$ being confirmed by the AC2 and AGC assays, respectively (Table 2). All 114 SDA-negative specimens were confirmed as negative using all four confirmatory tests.

\section{DISCUSSION}

This study set out to examine different approaches for confirming the $N$. gonorrhoeae status of $\mathrm{BD}$ ProbeTec residual clinical specimens. This is of particular relevance as in a recent survey it was highlighted that $61 \%$ of diagnostics laboratories in the UK currently are using this 
Table 2. Correlation between the SDA $N$. gonorrhoeae test and the confirmatory testing methods

\begin{tabular}{|c|c|c|c|c|c|c|}
\hline $\begin{array}{l}\text { Lab. one } \\
\text { SDA }\end{array}$ & porA gene & opa gene & AC2 & AGC & $\begin{array}{l}\text { No. of specimens } \\
\qquad(n=227)\end{array}$ & Consensus result \\
\hline NEG & NEG & NEG & NEG & NEG & 114 & NEG \\
\hline POS & POS & POS & POS & POS & 16 & POS \\
\hline POS & POS & POS & NEG & NEG & 62 & POS \\
\hline POS & NEG & POS & NEG & NEG & 1 & POS \\
\hline POS & POS & POS & POS & NEG & 8 & POS \\
\hline POS & POS & POS & NEG & POS & 11 & POS \\
\hline POS & POS & NEG & POS & POS & 3 & POS \\
\hline
\end{tabular}

platform and confirmatory testing is required if these laboratories are to comply with national $N$. gonorrhoeae NAAT guidelines (Benzie et al., 2010; Health Protection Agency, 2010; Dave \& Eastaway, 2007). It was determined that independent in-house real-time PCR assays, combined with appropriate DNA extraction facilities, are suitable confirmation tests for SDA assay specimens as high rates of correlation can be achieved. However, the use of the AC2 and the AGC tests cannot be reliably used to confirm residual urine specimens in SDA assay collection tubes.

The failure of both the AC2 and the AGC tests to confirm the $N$. gonorrhoeae status of the residual urine specimen is surprising and is probably due to the incompatibility of different platform chemistries. In previous studies, the AC2 assay has been demonstrated to have superior sensitivity and specificity to the SDA test and therefore should be a suitable assay for confirmation (Ota et al., 2009). Unfortunately, both the AC2 and AGC assays lack an internal control so it is not possible when using this confirmatory approach to monitor for inhibition, which may be caused by the unavoidable addition of invalidated collection buffers from other manufacturers. The results of this study reinforce the premise that when using $N$. gonorrhoeae NAATs, both the frontline assay and the laboratory's confirmatory testing strategy should be validated as a single protocol prior to introduction.

At the present time, there is a paucity of studies examining $N$. gonorrhoeae NAATs confirmatory testing strategies that could be undertaken within a primary diagnosis laboratory. In a large study conducted by Moncada et al. (2008), different approaches to $N$. gonorrhoeae NAATs testing were explored for the three main commercially available $N$. gonorrhoeae NAATs platforms, including repeat testing, confirmatory testing on an alternative platform and confirmatory testing on a secondary specimen from the same patient. Whilst the study was comprehensive and the protocol complex and as such numerous conclusions were drawn, it was found that the AC2 assay could confirm $85 \%$ of the SDA-positive specimens examined and the authors speculated that optimal NAAT performance may be obtained only with specimens collected in the transport medium designed for each specific NAAT. It is possible that the higher rates of confirmation achieved by Moncada et al. (2008) may be because a smaller volume of residual specimen $(100 \mu \mathrm{l})$ was added to the AC2 collection tube than in this study $(200 \mu \mathrm{l})$ or because a different range of specimen types were examined. The larger volume tested and the exclusive use of urine specimens in SDA assay transportation buffer may have led to greater inhibition in our study, which is not possible to monitor. Unfortunately, however, no standardized methodology for reliably confirming specimens between the different commercial NAATs has yet been published.

Another study examining two different strategies for confirming all SDA-positive specimens on the AC2 platform was also explored by Hardwick et al. (2009). The first approach involved the direct transfer of residual SDA assay transportation buffer into APTIMA collection tubes followed by testing; the second approach involved either (i) taking the original vaginal swab from the SDA assay collection tube and transferring to an APTIMA tube for a fixed period of time or (ii) splitting the original neat urine sample into an APTIMA tube, followed by testing on the AC2 platform. Such approaches yielded confirmation rates of $53.1 \%$ and $97.3 \%$, respectively. Clearly, from examining data obtained from both the Hardwick et al. (2009) study and indeed this study it can be concluded that it is not reliable to directly transfer the residual material from an SDA assay tube into an APTIMA tube and confirm it on the APTIMA platform. Whilst it may be possible to transfer a clinical swab from the SDA collection tube into the APTIMA tube and retest, such confirmatory testing with urine specimens is clearly impossible using this approach unless the laboratory has direct access to the raw sample.

The application of confirmatory testing when using $N$. gonorrhoeae NAATS in low prevalence settings has numerous benefits and should raise the positive predictive value of the test and reduce the chance of patients being misdiagnosed with gonorrhoea. However, in practice it can be difficult to achieve, as few laboratories have access to 
more than one N. gonorrhoeae NAAT testing platform, and, as this study has highlighted, even when laboratories do have access to more than one NAAT, the incompatibility of the different collection buffers makes confirmatory testing problematic. Whilst the application of well-validated inhouse real-time PCRs and robust DNA extraction methods has been demonstrated to be a suitable alternative for confirmatory testing, establishing such tests for diagnostic purposes in all primary laboratories would be a major undertaking. In reality, in-house confirmatory PCR services may be more suitable for larger diagnostics laboratories and/or specialist laboratories where demand for their use is high and quality control can be prioritized.

\section{REFERENCES}

Benzie, A., Alexander, S., Gill, N., Greene, L., Thomas, S. \& Ison, C. (2010). Gonococcal NAATs: what is the current state of play in England and Wales? Int J STD AIDS 21, 246-248.

Chen, C. Y., Chi, K. H., Alexander, S., Martin, I. M., Liu, H., Ison, C. A. \& Ballard, R. C. (2007). The molecular diagnosis of lymphogranuloma venereum: evaluation of a real-time multiplex polymerase chain reaction test using rectal and urethral specimens. Sex Transm Dis 34, 451-455.

Dave, J. \& Eastaway, A. (2007). Guidance on the Introduction of Molecular Testing for Neisseria gonorrhoeae in Diagnostic Laboratories. Health Protection Scotland. Available at: http://www.documents.hps. scot.nhs.uk/labs/sbstirl/naats-2007-08.pdf
Hardwick, R., Gopal Rao, G. \& Mallinson, H. (2009). Confirmation of BD ProbeTec Neisseria gonorrhoeae reactive samples by Gen-Probe APTIMA assays and culture. Sex Transm Infect 85, 24-26.

Health Protection Agency (2010). Guidance for Gonorrhoea Testing in England and Wales. http://www.bashh.org/news/478_2010-hpaguidance-on-gonorrhoea-testing

Ison, C. (2006). GC NAATs: is the time right? Sex Transm Infect 82, 515.

Moncada, J., Donegan, E. \& Schachter, J. (2008). Evaluation of CDCrecommended approaches for confirmatory testing of positive Neisseria gonorrhoeae nucleic acid amplification test results. J Clin Microbiol 46, 1614-1619.

Ota, K. V., Tamari, I. E., Smieja, M., Jamieson, F., Jones, K. E., Towns, L., Juzkiw, J. \& Richardson, S. E. (2009). Detection of Neisseria gonorrhoeae and Chlamydia trachomatis in pharyngeal and rectal specimens using the BD Probetec ET system, the Gen-Probe Aptima Combo 2 assay and culture. Sex Transm Infect 85, 182-186.

Palmer, H. M., Mallinson, H., Wood, R. L. \& Herring, A. J. (2003). Evaluation of the specificities of five DNA amplification methods for the detection of Neisseria gonorrhoeae. J Clin Microbiol 41, 835-837.

Tabrizi, S. N., Chen, S., Tapsall, J. \& Garland, S. M. (2005). Evaluation of opa-based real-time PCR for detection of Neisseria gonorrhoeae. Sex Transm Dis 32, 199-202.

Whiley, D. M. \& Sloots, T. P. (2005). Comparison of three in-house multiplex PCR assays for the detection of Neisseria gonorrhoeae and Chlamydia trachomatis using real-time and conventional detection methodologies. Pathology 37, 364-370.

Whiley, D. M., Tapsall, J. W. \& Sloots, T. P. (2006). Nucleic acid amplification testing for Neisseria gonorrhoeae: an ongoing challenge. J Mol Diagn 8, 3-15. 